\title{
Obstrucción maligna de la vena cava superior
}

\section{Malign obstruction of the superior vena cava}

M.A. Domínguez, A. Manterola, P. Romero, E. Martínez, F. Arias, E. Villafranca, M. Martínez

\section{RESUMEN}

En el presente trabajo se revisan los síndromes de la vena cava superior malignos, sus manifestaciones clínicas, relacionadas con las características anatómicas del compartimento por donde discurre la vena cava superior, los requerimientos diagnósticos para abordar el tratamiento en las mejores condiciones y el conjunto de medidas que deben adoptarse para su manejo.

Palabras clave. Vena cava superior. Obstrucción. Tumores malignos.

\begin{abstract}
The present paper offers a review of the malign syndromes of the superior vena cava, their clinical expressions related to the anatomical characteristics of the compartment where the superior vena cava runs, the diagnostic requirements for realising treatment under the best conditions and the ensemble of measures that must be adopted in dealing with this.

Key words. Superior vena cava. Obstruction.
\end{abstract} Malign tumours.

An. Sist. Sanit. Navar. 2004; 27 (Supl. 3): 99-107.

Servicio de Oncología Radioterápica. Hospital de Navarra. Pamplona.
Correspondencia:

Miguel Angel Domínguez Domínguez Servicio de Oncología Radioterápica

Hospital de Navarra

Irunlarrea, 3

31008 Pamplona

Tfno. 848422162

E-mail: ma.dominguez.dominguez@cfnavarra.es 


\section{INTRODUCCIÓN}

El síndrome de vena cava superior (SCVS) es una manifestación poco habitual de las enfermedades malignas, afectando a una minoría de pacientes en algún momento de su enfermedad. Se define por los signos y síntomas derivados de la obstrucción del flujo sanguíneo en la vena cava superior (VCS). Las neoplasias malignas dan lugar al $90 \%$ de los casos de SVCS y son en su mayoría carcinomas de pulmón ${ }^{1}$. Menos comúnmente el SVCS maligno está producido por linfomas, metástasis y tumores intratorácicos como mesoteliomas o timomas ${ }^{2}$.

\section{ANATOMÍA Y FISIOPATOLOGÍA}

Los síntomas y signos característicos del síndrome de la vena cava superior (SVCS) aparecen cuando este vaso de finas paredes es comprimido, invadido o trombosado por procesos que se desarrollan en el mediastino superior.

La VCS se extiende en el mediastino desde la unión de las venas innominadas derecha e izquierda hasta la aurícula derecha, en un trayecto de 6 a $8 \mathrm{~cm}$, los dos últimos dentro del saco pericárdico. $\mathrm{Su}$ anchura es de 1,5 a $2 \mathrm{~cm}$ y contiene sangre a baja presión.

Por sus características la VCS es vulnerable a cualquier proceso ocupante de espacio que se desarrolle en su vecindad. Además se encuentra completamente rodeada por cadenas ganglionares que drenan todas las estructuras de la cavidad torácica derecha y de la inferior izquierda.

Otras estructuras del mediastino, como el bronquio principal, el esófago o la médula espinal, pueden estar en riesgo por procesos que obstruyan la circulación por la VCS. La vena ácigos, principal vaso auxiliar de la VCS, que entra en ésta por su cara posterior, inmediatamente por encima de la reflexión pericárdica, y es la vía alternativa más importante a la circulación por la VCS, está también amenazada por estas patologías.

Las venas mamarias internas, las venas torácicas largas, las venas paraespinosas y la red de venas esofágicas aportan otros sistemas colaterales. Las venas subcutáne- as son también vías importantes y su dilatación en el cuello y tórax es un signo típico del SVCS.

A pesar de las vías colaterales la presión venosa en los casos de obstrucción de la VCS está casi siempre elevada en el compartimento superior. Se ha detectado hasta de 200 a $500 \mathrm{~cm}$ de $\mathrm{H}_{2} \mathrm{O}^{3}$.

La fisiopatología del síndrome está relacionada con la obstrucción del drenaje venoso de la parte superior del tórax, con el consiguiente incremento de la presión venosa que provoca la dilatación de las venas colaterales, plétora facial, edema conjuntival con o sin proptosis, y varios síntomas neurológicos centrales, como cefaleas, trastornos visuales y alteraciones en el estado de conciencia ${ }^{4}$.

La revisión de Roswit y col aporta un entendimiento completo de las características anatómicas y fisiopatológicas del síndrome $^{5}$. La vulnerabilidad de la VCS está relacionada con la fragilidad de las paredes de la vena y con la baja presión venosa. La VCS está encerrada en un apretado compartimento del mediastino anterosuperior, detrás del rígido esternón, al lado del bronquio principal derecho, y completamente rodeada por las cadenas de ganglios linfáticos que drenan todas las estructuras de la cavidad torácica derecha y la parte inferior de la izquierda. El principal vaso auxiliar, la vena ácigos, está también rodeado por cadenas linfáticas y amenazado en caso de aumento del volumen de los ganglios de las mismas. En consecuencia, sin una apropiada intervención terapéutica en esta encrucijada anatómica los daños por la obstrucción de la VCS pueden llevar a su trombosis irreversible, a lesiones en el SNC y a complicaciones pulmonares o medulares entre otras.

En la práctica corriente, la causa más frecuente de obstrucción de la VCS es el cáncer. Desde la llegada de los antibióticos, las enfermedades benignas dejaron de ser las causantes más frecuentes del SVCS. Actualmente los aneurismas sifilíticos y las mediastinitis tuberculosas como desencadenantes de SVCS son infrecuentes. El artículo de Schechter en 1954 indicaba que el $40 \%$ de 274 casos de SVCS eran debidos a la sífilis o a la tuberculosis, pero en la 
actualidad las enfermedades malignas son causantes de alrededor del $97 \%$ de todos los casos de $\mathrm{SCVS}^{6,7}$. La causa más frecuente no maligna de SCVS es el bocio, mientras que otras condiciones benignas causantes de SVCS incluyen las trombosis primarias de la vena cava superior, las pericarditis constrictivas y las mediastinitis esclerosantes idiopáticas.

En cuanto a las enfermedades malignas, el carcinoma de pulmón y los linfomas causan la inmensa mayoría de los SVCS. En una combinación de series, el $75 \%$ fueron debidos a carcinomas broncogénicos, el $15 \%$ a linfomas y el $7 \%$ a enfermedad metastásica?

El análisis de Pérez y col revela que el tipo más frecuente de carcinomas de pulmón es el carcinoma anaplásico de células pequeñas, causante del $46 \%$ de los casos de SVCS, seguido de los carcinomas epidermoides $^{8}$. La preponderancia de los carcinomas de células pequeñas es predecible dada la conocida tendencia de estos tumores a desarrollarse en localizaciones centrales y parahiliares. Entre los catorce pacientes con linfomas de esta serie, la distribución por subtipos histológicos resulta homogénea entre los clasificados como linfocíticos e histiocíticos.

La obstrucción resultante en el SCVS se puede producir como resultado de la compresión extrínseca de la VCS por metástasis ganglionares o por el tumor primario, o la pared del vaso puede resultar directamente infiltrada por el tumor. En los exámenes post-mortem en $1 / 3$ a $1 / 2$ de los pacientes con SVCS aparece trombosada la $\mathrm{VCS}^{8}$. De hecho, el fracaso en la reversión del síndrome se debe frecuentemente a la obstrucción trombótica de la vena.

\section{CURSO CLÍNICO}

Habitualmente el SCVS se establece insidiosamente y progresivamente aparecen los signos y síntomas característicos de la enfermedad ${ }^{9}$. La disnea es el síntoma más común, presente en el $63 \%$ de los casos. El $50 \%$ de los pacientes refieren sensación de plenitud en la cabeza y de hinchazón en el cuello. Hinchazón del tronco y de las extremidades superiores se aqueja con una frecuencia similar. Dolor toráci- co, tos y disfagia se refieren menos habitualmente (en alrededor del $20 \%$ de los casos cada uno de ellos) ${ }^{10}$.

En cuanto a los hallazgos clínicos y signos presentes en el SVCS, la ingurgitación de las venas del tórax y del cuello son los más frecuentes, seguidos de edema facial, taquipnea, plétora y cianosis. Estos síntomas y signos pueden agravarse al inclinarse hacia delante, agacharse o tumbarse y tienden a ser más severos cuando la VCS está obstruida por debajo de la entrada de la vena ácigos ${ }^{11}$.

\section{DIAGNÓSTICO}

El diagnóstico clínico es habitualmente aparente sin excesivas pruebas diagnósticas. Las radiografías de tórax muestran una masa en el mediastino superior en casi todos los pacientes (en el 75\% predominantemente en el lado derecho). Sólo el $16 \%$ de los pacientes presentan una radiografía de tórax normal. Las anomalías más frecuentes en las radiografías de tórax son ensanchamientos del mediastino superior y derrames pleurales ${ }^{12}$.

El TAC aporta información adicional sobre la VCS, sus tributarias y sobre otras estructuras mediastínicas como los bronquios y la médula espinal. Esta información adicional es necesaria para detectar la posible urgencia en la actuación para liberar la presión sobre estas estructuras. Además es un valor añadido del TAC (y de la RM) su capacidad para asistir a la planificación de la radioterapia delimitando la extensión del tumor.

La flebografía ofrece excelente información sobre la localización de la compresión y el estado de las colaterales.

En cuanto a la RM, su papel debe ser aún investigado aunque su aportación parece prometedora, máxime al tratarse de una prueba no invasiva.

La venografía aporta información para determinar si la obstrucción de la VCS es completa, permanece aún transitable o está estenótica por compresión extrínsica. Frecuentemente -en el $41 \%$ en la serie de Dyet y Moghissi- la vena está desplazada o infiltrada, pero no completamente obstruida por el tumor ${ }^{13}$. Un $19 \%$ tienen la com- 
presión de la VCS por debajo de la vena ácigos. La venografía se utiliza en la actualidad para evaluar las condiciones previas a la colocación de una malla en la luz de la vena. Aunque se ha preconizado que la venografía debe ser evitada por el riesgo de sangrado excesivo en el lugar de la punción, en la circunstancia que contemplamos, es decir la existencia de un aumento de la presión intraluminal en el territorio de la VCS, no se han publicado evidencias sobre este riesgo. Sin embargo, aunque una venografía puede confirmar el diagnóstico clínico y perfilar la anatomía del enclave, la prioridad debe ser para las pruebas que ayudan a establecer el diagnóstico histológico, salvo ante la decisión de realizar un procedimiento terapéutico intraluminal desobstructivo.

La venografía con tecnecio 99 es una alternativa mínimamente invasiva de obtener imágenes del sistema venoso. Aunque las imágenes obtenidas por este método no son de tanta calidad como las de las venografías con contraste, sirve para mostrar la permeabilidad del vaso y los patrones de flujo. La circulación colateral puede ser evaluada de una manera general por la venografía con radionucleido.

En el 58\% de 107 pacientes el SVCS se desarrolló antes de que el tumor primario causante fuera diagnosticado, según Schraufnagel y $\mathrm{col}^{14}$. Entre los procedimientos utilizados para el diagnóstico histológico la citología de esputo estableció el diagnóstico en casi el 50\% de los casos. El diagnóstico citológico es tan seguro como el basado en muestras de tejido en los carcinomas de células pequeñas. Por otra parte la broncoscopia aporta células para el diagnóstico en la mayoría de los carcinomas microcíticos, mientras que en los casos en los que existe derrame pleural una toracocentesis permite el diagnóstico en el $71 \%$. La biopsia de ganglios supraclaviculares, especialmente en el caso de palpación sospechosa, resulta positiva en dos tercios de los casos intentados.

La mediastinoscopia, finalmente, tiene una muy alta tasa de éxito en conseguir el diagnóstico histológico, y una tasa de complicaciones en torno al 5\%. Varios estudios, sobre la utilización de la mediasti- noscopia para pacientes en los que el diagnóstico histológico no pudo ser establecido por otro procedimiento menos invasivo, han confirmado la seguridad y eficacia de la mediastinoscopia, sin mortalidad perioperatoria $^{15,16}$.

Procedimientos invasivos para obtener el diagnóstico histológico deben llevarse a cabo si no se detecta tejido tumoral superficial del que obtener material para estudio patológico: biopsias por broncoscopia, esofagoscopia, mediastinoscopia o toracotomía exploradora. Tales procedimientos deben ser acometidos siempre en los casos de diagnóstico histológico desconocido, salvo que sea necesaria una intervención terapéutica inmediata ante una situación vital comprometida. Siempre que el tratamiento y el pronóstico dependan del conocimiento del tipo histológico y de la localización anatómica del tumor primario, el diagnóstico definitivo es necesario.

Con la citología de esputo, la broncoscopia y las citologías y biopsias obtenidas con este procedimiento, y con la biopsia de adenopatías supraclaviculares, se consigue el diagnóstico en el $70 \%$ de los casos de SVCS. Cuando resulta finalmente necesaria, la toracotomía es casi siempre eficaz en obtener tejido para diagnóstico.

La biopsia con aguja fina guiada por TAC es una técnica emergente como una alternativa efectiva, en algunos casos, a la biopsia abierta o a la mediastinoscopia. La aterectomía transluminal se ha utilizado también con éxito diagnóstico ocasionalmente.

La opinión tradicional de que los procedimientos diagnósticos invasivos llevan consigo importantes riesgos para los pacientes con SVCS, especialmente de sangrado, ha sido revisada y desestimada en los últimos tiempos. Ahman examinó los resultados en 843 pacientes sometidos a procedimientos diagnósticos invasivos o semiinvasivos y encontró que sólo diez presentaron complicaciones y ninguna de ellas fatales ${ }^{17}$. En sus conclusiones rechazan la existencia de evidencias para sugerir que procedimientos como venografías, toracotomías, broncoscopios, mediastinoscopias y biopsias ganglionares supraclaviculares, supongan un riesgo excesivo 
para los pacientes con SVCS17. En 163 pacientes tratados en el Memorial SloanKettering Cancer Center por tumores voluminosos en el mediastino anterior, 44 fueron sometidos a anestesia general, sin mortalidad operatoria y con sólo cuatro intubaciones prolongadas ${ }^{18}$.

\section{TRATAMIENTO}

Aliviar y curar, como en todos los procesos malignos y no malignos, son los objetivos en el manejo de los pacientes con SCVS de etiología maligna. La presencia de obstrucción de la VCS en un paciente con cáncer no es por sí misma motivo para modificar la posible intención curativa del tratamiento. Máxime si tenemos en cuenta que el $50 \%$ de los pacientes con SVCS padecen carcinoma de pulmón anaplásico de células pequeñas (CPCP), linfomas no Hodgkin (LNH) o tumores germinales, todos ellos potencialmente curables aún en los casos de enfermedad diseminada. En este sentido, se debe mantener el criterio de decidir el tratamiento en función del tipo histológico de la enfermedad maligna y de la extensión de la misma. El pronóstico en los pacientes con SVCS depende del de la enfermedad de base y de los factores pronósticos reconocidos para la misma, que siempre incluyen la estadificación y en ningún caso la presencia o no de obstrucción de la VCS.

Cuando la intención es exclusivamente paliar los síntomas y signos relacionados con la obstrucción de la VCS o cuando la situación derivada de este bloqueo es una urgencia vital, la apertura directa de la vena debe considerarse. En este sentido las nuevas técnicas para colocación de mallas metálicas autoexpansibles endovascularmente y de angioplastia con balón, con posible trombolisis asociada, pueden aportar alivio rápido, mientras se establece el tratamiento específico adecuado $^{19,20}$.

\section{Radioterapia}

La radioterapia es el tratamiento antitumoral de elección en los casos de tumores no especialmente quimiosensibles -habitualmente CPNCP-. En estos casos, aunque el pronóstico sea generalmente pobre, dependiente de los factores pronósticos negativos que habitualmente acompañan a estos tumores, la probabilidad de mejorar los signos y síntomas del SVCS con radioterapia es alta. No se apartan tampoco estos casos de la regla de oro de ofrecer a los pacientes el mejor tratamiento específico antineoplásico en función de la extensión de la enfermedad y de las condiciones del paciente: sea radioterapia o quimioradioterapia concomitante o combinaciones secuenciales de las dos armas terapéuticas.

Aunque algunas revisiones indican que una malla intravascular puede sustituir a la radioterapia cuando su papel es exclusivamente paliativo, frecuentemente la obstrucción de la VCS se acompaña de amenazas ya instauradas o potenciales de riesgo incluso vital, como compresiones de la vía aérea al nivel de la tráquea o de bronquios principales, de la médula espinal o del esófago que requieren el concurso de entrada o posterior de la radioterapia. La radioterapia es, por tanto, una opción a considerar de pronta implementación en la mayoría de estos casos de SVCS. Debe ser utilizada en los casos que no se consideren tributarios de procedimientos que permitan el diagnóstico histológico, bien por soportar una situación gravemente deteriorada o por fracasos repetidos en las maniobras invasivas tendentes a obtener tejido para estudio histológico, sin perder de vista que el SVCS raramente se presenta como una urgencia absoluta y que para el alivio rápido de los signos y síntomas exclusivamente debidos a la obstrucción de la VCS se puede contar con la colocación de mallas metálicas intravasculares.

Ningún dato claro sirve para sostener un fraccionamiento de la dosis total de irradiación especial para los casos de SCVS, máxime en el momento actual de consenso acerca de la rareza de presentación del SVCS como una urgencia real. Ante esta última situación clínica cabría plantearse algún esquema de irradiación modificado, en relación con los considerados óptimos para los CPNCP y otros tumores no quimiosensibles, para potenciar la posibilidad de una respuesta rápida. Estos esquemas modificados se caracterizan por la administración de dos a cuatro fracciones de 3 a 4 Gy, segui- 
das de las necesarias para alcanzar la dosis total requerida, hasta 60-66 Gy, cuando la intención es curativa.

En los supuestos de tratamientos radioterápicos paliativos para pacientes con SVCS las dosis totales de irradiación se mueven en el entorno de 30 Gy y el fraccionamiento entre 3 y 4 Gy. En el ensayo promovido por Rogrigues y col una fracción semanal de 8 Gy hasta alcanzar los 24 Gy se comparó con una dosis total de 16 Gy en una semana, en dos fracciones de $8 \mathrm{~Gy}^{21}$. La disfagia transitoria fue el efecto secundario más relevante en casi en $50 \%$ de los pacientes, en los dos brazos. La tasa de respuestas completas fue del $56 \%$ en los pacientes tratados con 24 Gy frente al $28 \%$ en los tratados con 16 Gy. Además en el brazo de 24 Gy se obtuvo un $40 \%$ de respuestas parciales, lo que completa un $96 \%$ de respuestas con un esquema similar a los 30 Gy en 10 fracciones utilizados habitualmente en los regímenes de irradiación paliativa. El tiempo medio hasta la recidiva del SVCS y la mediana de supervivencia también fue superior en el brazo de dosis más alta.

Por último, se considera probable, a la luz de hallazgos utilizando venografías seriadas y autopsias, que la mejoría sintomática aportada por la radioterapia no se debe siempre al restablecimiento de la permeabilidad venosa por la VCS, sino también a la facilitación para la formación de colaterales que aportaría la descompresión producida en el mediastino por la radioterapia.

El volumen a irradiar en los casos de carcinomas de pulmón con SVCS debe abarcar, además del volumen tumoral con un margen tridimensional adecuado para asegurar la dosis prescrita en las áreas afectadas clínica y subclínicamente en el mediastino, las regiones supraclaviculares mediales, para reducir la alta tasa de recaídas ganglionares supraclaviculares que aparecen cuando estas regiones no se incluyen en el volumen de tratamiento.

\section{Tratamiento sistémico}

La quimioterapia, sola o combinada con la radioterapia, es el tratamiento de elección para los CPCP. Aunque ambas modalidades terapéuticas son altamente eficaces en resolver con rapidez el SVCS en estos pacientes, la prioridad para la quimioterapia basada en el cisplatino y el etopósido deriva de su papel primordial en los tratamientos potencialmente curativos, y en los mejores tratamientos paliativos, para la mayoría de los CPCP.

Las tasas de respuesta a la quimioterapia, y a la radioterapia, son frecuentemente superiores al $90 \%$ en los SCVS secundarios a CPCP, y en los casos de recaída después de quimioterapia las tasas de respuesta a la radioterapia o a más quimioterapia siguen siendo significativas, lo suficiente como para considerar, en términos generales, como una buena alternativa el retratamiento de estos pacientes ${ }^{22}$.

Cuando se administra quimioterapia en pacientes con CPCP se deben evitar las venas de los brazos, para lo que la circulación venosa de las extremidades inferiores ofrece una sencilla alternativa.

También en los LNH el tratamiento sistémico con quimioterapia es la mejor opción terapéutica para los pacientes con SCVS. Aunque la radioterapia es tan eficaz como la quimioterapia en aliviar rápidamente los signos y síntomas de la obstrucción de la VCS, ésta ofrece la eficacia local y sistémica necesaria para garantizar las mejores opciones curativas.

Ocho de 22 pacientes (81\%), en la serie del M. D. Anderson Cancer Center de linfomas de células grandes con SCVS alcanzaron la remisión completa ${ }^{23}$. Los investigadores recomiendan también en estos casos que el tratamiento se base en el diagnóstico histológico, conviniendo que el SCVS secundario a LNH rara vez es una emergencia que dificulte la puesta en marcha de los procedimientos necesarios para obtener el diagnóstico histológico y realizar un estudio de extensión adecuado. Sin embargo, la obstrucción traqueal acompaña al SCVS preferentemente en los pacientes con LNH, y en estos casos la situación debe manejarse como una emergencia absoluta.

La linfografía, actualmente en desuso, debe ser evitada en estos pacientes para prevenir la embolización del contraste y el fallo respiratorio subsiguiente. La utilización de radioterapia de consolidación se 
debe ajustar a los criterios generales de tratamiento de los linfomas, independientemente de la presencia de síntomas y signos de obstrucción de la VCS, salvo en los raros casos de urgencia vital.

\section{Mallas autoexpansibles y angioplastia}

La angioplastia percutánea con balón y la colocación endoluminal de mallas metálicas autoexpandibles se utilizan, con frecuencia creciente a medida que las técnicas están disponibles en más centros, para abrir la luz del vaso y mantener el flujo a través del mismo en casos de obstrucción maligna de la VCS. El rápido (24-72h) y persistente control de los síntomas del síndrome en la mayoría de los casos, con una baja tasa de complicaciones, las convierte en medidas terapéuticas paliativas especialmente importantes en pacientes cuya esperanza de vida está severamente reducida y sin posibilidades de beneficiarse de los tratamientos etiológicos.

La rapidez y eficacia sintomática de la colocación endoluminal de mallas autoexpandibles en los tramos obstruidos de la VCS convierten esta técnica en un procedimiento de valor también como complemento a los tratamientos específicos antitumorales indicados en los SCVS malignos. De manera que la selección de casos exclusivamente para tratamientos endoluminales o para apertura rápida de la VCS seguida de la radioterapia o quimio-radioterapia necesarias para administrar el tratamiento potencialmente curativo o el mejor tratamiento paliativo posible, o para resolver la reaparición o resolución incompleta del síndrome después de quimioterapia y/o radioterapia, forma parte del mejor manejo posible del SCVS maligno. Sería un error, por el contrario, considerar estas técnicas como sustitutivos sistemáticas de los tratamientos antitumorales, a pesar de resultados de estudios comparativos no controlados, que no tienen en cuenta ni las múltiples variables pronósticas que concurren en las series de pacientes con obstrucción maligna de la VCS, ni la falta de una escala mundialmente aceptada para cuantificar la gravedad de la obstrucción maligna de la VCS.
La trombolisis con agentes fibrinolíticos es a menudo parte integral de las técnicas intraluminales, como necesidad derivada de deshacer el trombo localizado en la VCS en los casos en que existe.

Finalmente, las poco frecuentes complicaciones después de los procedimientos endovasculares que nos ocupan incluyen la migración o descolocación de la malla, dolor durante el inflado del balón, edema pulmonar agudo cardiogénico, taponamiento cardíaco, desgarros en la vena o elevación transitoria del diafragma.

\section{Bypass quirúrgico}

En pacientes con SVCS maligno el bypass quirúrgico puede ser intentado exclusivamente cuando las maniobras anteriores endoluminales, asociadas a radioterapia o radioquimioterapia han fallado, y el pronóstico del paciente lo permite. Se han utilizado injertos autólogos o prótesis de Dacron o de politetrafluoroetileno expandido, aunque la experiencia en casos de obstrucción maligna de la VCS es escasa.

\section{Trombolisis}

La trombolisis es una parte importante de los procedimientos endoluminales completos -angioplastia con balón seguida de colocación de malla autoexpandiblecuando se detecta la presencia de un trombo en la VCS. En la experiencia de la Cleveland Clinic la uroquinasa ha resultado más efectiva que la estreptoquinasa, y el retraso en iniciar el tratamiento de más de 5 días desde el comienzo de los síntomas resultó en fracaso terapéutico ${ }^{24}$.

\section{Medidas generales}

Diuréticos y corticoesteroides se utilizan frecuentemente en el manejo general de los pacientes con SVCS. Los diuréticos se han propuesto para mejorar el edema, pero efectos adversos derivados del incremento de fenómenos embólicos por deshidratación han llevado a restringir su uso.

Los esteroides forman parte del manejo estándar del SVCS a pesar de la ausencia de evidencia para mantener su uso -6 a 10 mg de dexametasona, oral o i.v., cada 6 
horas-. En ausencia de evidentes problemas por edema radioinducido, o fuera de su uso en SCVS producidos por linfomas, un ensayo randomizado comparando el uso rutinario de esteroides con un uso electivo está justificado ${ }^{2}$. $\mathrm{Si}$, en cualquier caso, se utilizan esteroides, deben utilizarse sólo durante un tiempo limitado altas dosis.

Medidas como el reposo con la cabeza elevada y la oxigenoterapia se recomiendan sistemáticamente porque alivian los síntomas del paciente como consecuencia de mejorar el gasto cardíaco y la presión venosa.

\section{CONCLUSIONES}

En el SCVS sin un claro diagnóstico etiológico deben darse los pasos necesarios para establecer el diagnóstico de la enfermedad de base antes de intentar un tratamiento antineoplásico. Cuando exista la necesidad de administrar terapias antineoplásicas dirigidas a la enfermedad maligna de base, sea para maximizar la supervivencia o para mejorar y mantener la calidad de vida, la quimioterapia y/o la radioterapia constituyen la base del tratamiento, como en pacientes con enfermedades malignas avanzadas sin SVCS.

La colocación de mallas intraluminales en tumores altamente quimiosensibles -CPCP, linfomas o tumores germinalesdebe considerarse ante la persistencia del SVCS o la parición de una recidiva.

En los casos de CPNCP, y de otros tumores de limitada quimiosensibilidad, el uso de procedimientos intraluminales con mallas autoexpandibles pueden asociarse en caso de recidiva o persistencia del síndrome a un tratamiento de inicio sólo específico -radioterápico o radio-quimioterápico- (adoptando medidas para un diagnóstico temprano de la recaída), pero también pueden utilizarse desde el comienzo del tratamiento.

En los raros casos en los que el SVCS representa una emergencia absoluta, habitualmente como consecuencia de la obstrucción de la vía aérea por el mismo proceso, la radioterapia puede ser necesaria inmediatamente, incluso sin un diagnóstico histológico establecido.

\section{BIBLIOGRAFÍA}

1. Ostler PJ, Clarke DP, WatKinson AF, Gaze MN Superior vena cava obstruction: a modern management strategy. Clin Oncol 1997; 9: 83-89.

2. Rowell NP, GleEson FV. Steroids, Radiotherapy, Chemotherapy and Stents for Superior Vena Caval Obstruccion in Carcinoma of the bronchus: a Systematic Review. Clin Oncol 2002; 14: 338-351.

3. JoAchim Y. Superior Vena Cava Síndrome. En: Cancer: Principles and Practice of Oncology, De Vita VT, Hellman S and Rosenberg A. Ed: Lippincott Williams \& Wilkins. 2001, pp.

4. AbNer A. Approch to the patient who presents with superior vena cava obstrucción. Chest 1993; 103 (Supl 4): 394S$397 \mathrm{~S}$

5. Roswit B, Kaplan G, Jacobson HG. The superior vena cava syndrome in bronchogenic carcinoma. Radiology 1953; 61: 722 .

6. SCHECHTER MM. The superior vena cava síndrome. Am J Med Sci 1954; 227: 46-56.

7. LOKICH JJ, GOODMAN RL. Superior vena cava síndrome. JAMA 1975; 231: 58-61.

8. Pérez CA, Presant CA, Amburg AL. Management of superior vena cava syndrome. Semin Oncol 1978; 5: 123-134.

9. Rashmi A, Fulvia M, VANDER Els NJ. Respiratory emergencies. Semin Oncol 2000; 27: 256-269.

10. Bell DR, Woods RL, LEVI JA. Superior vena caval obstruction: a 10-year experience. Med J Aust 1986; 145: 566-568.

11. StanFord W, Jolles H, Ell S, Chiu LC. Superior vena cava obstruction: a venographic classification. Am J Roentgenol 1987; 148: 259-262.

12. Parish JM, MarschKe RF, Dines DE, LeE RE. Etiologic considerations in superior vena cava syndrome. Mayo Clin Proc 1981; 56: 407-413.

13. Dyet JF, Moghissi K. Role of venography in assessing patients with superior vena cava obstruction caused by bronchial carcinoma for bypass operations. Thorax 1980; 35: 628630.

14. Schraufnagel DE, Hill R, Leech JA, Pare JA. Superior vena caval obstruction. Is it an emergency? Am J Med 1981; 70: 1169-1174.

15. Mineo TC, Ambrogi V, Nofroni I, Pistolese C. Mediastinoscopy in superior vena cava obstruction: analysis of 80 consecutive patients. Ann Thorac Surg 1999; 68: 223-226. 
16. JAHANGIRI M, GoldSTRAW P. The role of mediastinoscopy in superior vena caval obstruction. Ann Thorac Surg 1995; 59: 453455.

17. AHMANN FR. A reassessment of the clinical implications of the superior vena cava syndrome. J Clin Oncol 1984; 2: 961-969.

18. FERRARI LR, BEDFORD RF. General anesthesia prior to the treatment of anterior mediastinal masses in pediatric cancer patients. Anesthesiology 1990; 72: 991-995.

19. Urroticoechea A, Mesia R, Domínguez J, Falo C, Escalante E, Montes A et al. Treatment of malignant superior vena cava síndrome by endovascular stent insertion. Experience on 52 patients with lung cancer. Lung Cancer 2004; 43: 209-214.

20. Greillier L, Barlesi F, Doddoli C, DuRiex O, TORRE JP, JIMÉNEZ C et al. Vascular estenting for palliation of superior vena cava obstruccion in non-small lung cancer patients: A Future standard procedure? Respiration 2004; 71: 178-183.

21. Rodrigues CI, NJO KH, KarIM AB. Hypofractionated radiation therapy in the treatment of superior vena cava syndrome. Lung Cancer 1993; 10: 221-228.

22. Chan RH, DAR AR, Yu E, STITT LW, Whiston F, TRUONG $\mathrm{P}$ et al. Superior vena cava obstruction in small-cell lung cancer. Int J Radiat Oncol Biol Phys 1997; 38: 513-520.

23. PÉrez-Soler R, McLaughlin P, Velasquez WS, Hagemeister FB, Zornoza J, Manning JT et al. Clinical features and results of management of superior vena cava syndrome secondary to lymphoma. J Clin Oncol 1984; 2: 260-266.

24. GRAy BH, OLIN JW, GRadyr RA, Young JR, BARTHOLOMEW JR, RUSCHHAUPT WF. Safety and efficacy of thrombolytic therapy for superior vena cava syndrome. Chest 1991; 99: 54-59. 